\title{
High frequency of E148Q sequence variation in children with familial Mediterranean fever in southeast Turkey
}

\author{
Ünal Uluca, Assist. Prof., ${ }^{a}$ Aydin Ece, Prof. Dr., ${ }^{b}$ Velat Şen, Assist. Prof., \\ Salih Coşkun, Assist. Prof., ', Ali Güneş, ", Assist. Prof., Servet Yel, Assist. Prof., ${ }^{a}$ \\ Illhan Tan, Assist. Prof., ${ }^{a}$ Müsemma Karabel, Assoc. Prof., ${ }^{a}$ and Cahit Şahin, M. D. ${ }^{a}$
}

\begin{abstract}
Objective: The aim of this study was to investigate the spectrum of Mediterranean fever $(M E F V)$ gene mutations and genotypephenotype correlation in children with familial Mediterranean fever (FMF) in southeast Turkey. Methods: A total of 507 children (274 females) with FMF and MEFV gene mutation(s) were included. A 15-year retrospective evaluation was conducted; parameters analyzed were: age, sex, age at symptoms onset, age at FMF diagnosis, delay between symptoms onset and diagnosis, FMF attack symptoms, and response to colchicine. Disease severity scores were calculated and $M E F V$ mutation analysis was performed via real-time PCR for the 6 most frequent mutations. Children with comorbid diseases or tested negative for MEFV gene mutations were excluded to provide homogeneity.
\end{abstract}

Results: A family history of FMF was found in $60.2 \%(n=305)$ of patients. The most common symptoms reported for FMF attacks were abdominal pain $(98.0 \%)$, fever $(93.9 \%)$ and arthralgia $(47.3 \%) ; 75.0 \%$ of patients $(n=380)$ were heterozygous, $14.2 \%$ were homozygous $(n=72)$ and $10.8 \%$ were compound heterozygous $(\mathrm{n}=55)$. The following MEFV gene mutation alleles were identified: E148Q (40.1\%), M694V (25.9\%), V726A (15.8\%), R761H (7.4\%), M680I $(6.8 \%)$, and P369S (4.1\%). The M694V subgroup had the lowest mean age of disease onset and the highest mean disease severity score, whereas the E148Q group had later mean disease onset and the lowest mean disease severity score $(p<0.05)$. Conclusion: The highest E148Q mutation frequency and milder disease in the course of FMF in ourstudy population may be due to geographic and ethnic background dissimilarities of southeast Turkey.

Key words: familial mediterranean fever, genotype, phenotype, mutation, southeast Turkey.

http:/ / dx.doi.org/10.5546/aap.2015.eng.133

\section{INTRODUCTION}

Familial Mediterranean fever (FMF) is an autosomal recessively inherited disease characterized by recurrent episodes of fever accompanied by serositis attacks involving the peritoneum, synovia and pleura. ${ }^{1,2} \mathrm{FMF}$ patients frequently experience their first attack in childhood.

Genetic mutations of the gene leading to FMF are located at the short arm of chromosome 16. This gene encodes a protein called pyrin which prevents excessive leukocyte migration to sites of inflammation and that is exclusively expressed in granulocytes, monocytes, dendritic cells and serosal and synovial fibroblasts. ${ }^{3-5}$ Pyrin participates in apoptosis, cell recruitment and activation of procaspase-1, also known as interleukin 1-beta (IL-1 ) converting enzyme with associated processing and secretion of IL-1 and IL-8. Although the clear effect of pyrin and the molecular mechanism of MEFV mutations remain controversial, recent data supports the hypothesis that FMF is caused by loss-of-function mutations leading to increased IL-1 activation and accentuated immune activation which results in serositis including peritonitis, pleuritic and synovitis. ${ }^{6}$

The most common complication of untreated FMF is serum amyloid A deposition in various organs, especially in the kidneys. FMF is treated with colchicine to prevent future attacks and renal amyloidosis, which may lead to the development of nephrotic syndrome and eventual chronic renal insufficiency. ${ }^{1-3}$

Although diagnosis of FMF is primarily clinical, it is possible to confirm it by performing a $M E F V$ gene mutation analysis. ${ }^{4,5}$ In the Middle East more than $85 \%$ of FMF patients have one of the following MEFV mutations: M694V, M694I, M680I, 
V726A, and E148Q. ${ }^{2}$ The M694V mutation was reported to have a relatively severe phenotype, whereas V726A and E148Q have been reported with a milder disease course. ${ }^{1-3,7}$

FMF is more prevalent among certain ethnic groups such as the Turks, Arabs, Jews and Armenians. ${ }^{8}$ Turkey is one of the few countries in the world having high FMF frequency with the estimated prevalence of $1: 400$ to $1: 1000 .^{2,7}$ Gene sequence and phenotypic characteristics of FMF patients change according to geographic regions, which include people from certain ethnic origins both in Turkey and in the World. ${ }^{1,2,7-17}$ Unfortunately, there is a paucity of studies that have investigated the genotypic and phenotypic variations of FMF patients in southeast Turkey. ${ }^{3}$ Therefore, we aimed to investigate the genotypic and phenotypic characteristics of children with FMF in southeastern Turkey.

\section{MATERIALS AND METHODS}

Children diagnosed as positive for MEFV gene mutations and receiving medical care from Dicle University Hospital Department of Pediatric Nephrology and Rheumatology unit between 1998 and 2013 were enrolled. FMF diagnosis was confirmed according to the Tel-Hashomer criteria. ${ }^{4}$ A retrospective evaluation of hospital records was conducted to determine patients'age at diagnosis, sex, previous symptoms, FMF family history, parental consanguinity, MEFV genotype, duration of FMF attacks, and response to colchicine. The diagnosis of an FMF attack was done based on clinical features, including fever $\left(>38^{\circ} \mathrm{C}\right)$, abdominal pain and / or chest pain lasting 6-72 hours, skin rash or arthritis and laboratory findings including high erythrocyte sedimentation rate and C-reactive protein. Renal biopsy was performed in patients with nephrotic syndrome (24 hour urinary protein $>40 \mathrm{mg} / \mathrm{m}^{2} / \mathrm{h}$, hypoalbuminemia, edema and hypercholesterolemia). Renal amyloidosis was diagnosed in renal biopsy specimen based on existence of the amorphous pink amyloid deposits around the arteries, in the interstitium, or in the glomeruli with hematoxylin \& eosin stain and demonstrating a Congo red stain of the pink material. Complete response to colchicine was defined as complete control of the clinical manifestations and normalization of acute phase reactants; incomplete response was defined as persistence of some clinical manifestations and/ or some elevated acute phase reactants, and no response as no improvement in attack frequency and/or severity of the disease despite colchicine treatment. ${ }^{19}$

Exclusion criteria were having another diagnosis besides. Disease severity was scored with criteria adapted from Pras et al. ${ }^{18}$ by Ozen et al. ${ }^{19}$ A score ranging from 3-5 was considered mild disease, between 6-9 points was moderate disease, and greater than 9 points was designated as severe disease. ${ }^{19}$ The study protocol was approved by the Dicle University Hospital Ethics Committee.

\section{DNA extraction and $M E F V$ gene analysis}

The MEFV mutation analysis was performed via real-time PCR for the 6 most frequent mutations. Genomic DNA was extracted from $2 \mathrm{~mL}$ of peripheral venous blood samples collected in tubes containing EDTA (ethylenediaminetetraacetic acid). The genomic DNA was isolated using a Nucleospin Blood Kit (Qiagen-GmbH, Germany) following the manufacturer's protocol. The DNA concentration was determined using a NanoDrop spectrophotometer (Thermo Scientific, USA). The 6 most common mutations located in exon 2 (E148Q) and exon 10 (M694I, M694V, M680I, V726A and R761H) of the MEFV gene were determined by real-time PCR on a ABI Prism 7500 Fast (Applied Biosystems, California, USA) according to manufacturer's instructions (Dr. Zeydanli FMF type 1 mutation analysis system, Ankara, Turkey).

\section{Statistical analysis}

Statistical analyses were performed by using SPSS version 18.0 software (SPSS Inc., Chicago, IL, USA). Student's t-test was utilized to make comparisons between two independent groups of normally distributed numerical data. Comparisons of data that not normally distributed were done by the Mann-Whitney U-test or the Kruskal-Wallis test. Categorical variables were evaluated by Chi-squared test. A $p$-value less than 0.05 was accepted as statistically significant.

\section{RESULTS \\ Demographics}

The demographic and clinical characteristics of FMF subjects are outlined in Table 1. The study enrolled 507 children of 339 unrelated families; $54 \%$ were female and the mean age was $8.6 \pm 3.7$ years. There was a mean delay in diagnosis of FMF for $2.3 \pm 2.2$ years (Table 1). There were no 
differences in mean age, age of symptoms onset, age at diagnosis, delay between symptoms onset and diagnosis, and disease severity scoring between female and male patients ( $p>0.05$, data not shown).

\section{Clinical features}

A family history of FMF was documented in $60.2 \%$ of patients. Of a positive FMF family history, $49.8 \%$ only had one affected family member $(n=152), 16.6 \%$ had two affected family members $(n=84)$, and $13.6 \%$ had three or more family members with FMF $(n=69)$. Consanguineous marriages were found in $41.4 \%$ of parents $(n=201)$ (Table 1$)$.

Most common FMF attack symptoms were abdominal pain $(98.0 \%)$, fever $(93.9 \%)$, and arthralgia $(47.3 \%)$. The mean disease severity score was 6.1 (range 3-12) in 389 patients. The symptoms and disease severity score, attack duration and attack pattern of 452 FMF children are given at Table 2. Moderate disease is the most frequent characteristics of patients. Severe disease, attack duration more than 72 hours and more than 4 attacks per month were the least frequent features of the FMF patients (Table 2).

Regular colchicine use was found in $73.9 \%$ of patients, intermittent colchicine use in $22.4 \%$ of patients, and non-compliance was demonstrated in $3.6 \%$ of patients. Complete response to treatment with resolution of symptoms was

TABLE 1. Demographic and some clinical characteristics of the patients (N 389)

\begin{tabular}{lc}
\hline & n (\%) or Mean \pm SD (range) \\
\hline Age, years & $8.6 \pm 3.7(1-17.5)$ \\
Female / Male & $274 / 233$ \\
Parental consanguinity & $210(41.4)$ \\
Family history of FMF & $305(60.2)$ \\
Age at onset, years & $5.9 \pm 3.4(1-16.3)$ \\
Age at diagnosis, years & $8.3 \pm 3.4(1-16.6)$ \\
Delay in diagnosis, years & $2.3 \pm 2.2(0-12)$ \\
Follow up period, years & $7.4 \pm 3.5(0.5-12)$ \\
Disease severity score & $6.1 \pm 1.9(3-12)$ \\
Abdominal pain & $497(98.0)$ \\
Fever & $476(93.9)$ \\
Arthralgia & $240(47.3)$ \\
Myalgia & $120(23.7)$ \\
Arthritis & $80(15.8)$ \\
Chest pain & $66(13.8)$ \\
Erysipelas-like erythema & $59(11.6)$ \\
\hline
\end{tabular}

SD: Standard deviation. achieved in $68.3 \%$, decreased disease severity in $25.7 \%$, and no response in $6.0 \%$ of patients. Renal amyloidosis developed in $1.4 \%(n=7)$. Five of the patients with renal amyloidosis were homozygous for the M694V mutation and two of them were compound heterozygous with M694V/V726A and M694V/M680I mutations. Two of these patients with amyloidosis were phenotype II FMF patients.

\section{Patient genotypes}

The most frequently identified independent MEFV mutation was E148Q in $40.1 \%$ of patients followed by M694V in $25.9 \%$ and V726A in $15.8 \%$ (Table 3). The most common heterozygous genotype was E148Q/Null in 36.3\% of patients, $12.0 \%$ of patients demonstrated the V726A/Null genotype, and another $12.0 \%$ had the M694V/ Null genotype. The most widespread doublemutant genotypes detected were M694V/ M694V at $7.7 \%$ and E148Q/E148Q at 3.7\% (Table 3).

TABLE 2. Disease severity score, attack duration and attack pattern of 452 FMF children

\begin{tabular}{lc}
\hline & N (\%) \\
\hline Disease severity & $187(41.4)$ \\
Mild & $242(53.5)$ \\
Moderate & $23(5.1)$ \\
Severe & \\
\hline Attack duration (hours) & $88(19.5)$ \\
$<12$ & $68(15.0)$ \\
$12-24$ & $140(31.0)$ \\
$24-48$ & $120(26.5)$ \\
$48-72$ & $36(8.0)$ \\
$>72$ & \\
\hline Attack frequency (per month) & $88(19.5)$ \\
Random & $98(20.8)$ \\
$<1$ attack & $159(35.2)$ \\
$1-2$ attack & $64(14.2)$ \\
$3-4$ attack & $47(10.3)$ \\
$>4$ attack &
\end{tabular}

TABLE 3. The distribution of MEFV gene mutations in the study group

\begin{tabular}{lc} 
Mutation & $\begin{array}{c}\text { Number (\%) of independent alleles* } \\
(\mathbf{n}=634)\end{array}$ \\
\hline E148Q & $254(40.1)$ \\
M694V & $164(25.9)$ \\
V726A & $100(15.8)$ \\
R761H & $47(7.4)$ \\
M680I & $43(6.8)$ \\
P369S & $26(4.1)$ \\
\hline
\end{tabular}

*Totally 380 alleles were null. 
A total of $75.0 \%$ of patients $(n=380)$ were heterozygous, $14.2 \%$ of patients $(n=72)$ were homozygous, and $55(10.8 \%)$ were compound heterozygous (Tables 4, 5).

No significant differences were found between mean age $(p=0.66)$ and age at diagnosis $(p=0.72)$ between homozygous, compound heterozygous and heterozygote patient groups (data not shown). However, homozygous patients did have a significantly lower mean age at symptom onset when compared with the heterozygote group at $4.2 \pm 2.7$ years versus $6.3 \pm 3.5$ years, respectively $(p=0.005)$. There were significant differences in mean disease severity scores between homozygous $(6.9 \pm 2.0$ points) when compared to compound heterozygous

TABLE 4. MEFV genotypes in pediatric patients with familial Mediterranean fever

\begin{tabular}{lccc}
\hline \multicolumn{3}{c}{ MEFV genotype (n=507) } \\
\hline One allele & Number (\%) & Two alleles & Number (\%) \\
\hline E148Q / - & $184(36.3)$ & M694V / M694V & $39(7.7)$ \\
V726A / - & $72(14.2)$ & E148Q / E148Q & $19(3.7)$ \\
M694V / - & $61(12.0)$ & M694V / V726A & $14(2.8)$ \\
R761H / - & $34(6.7)$ & M680I / M680I & $12(2.4)$ \\
M680I / - & $17(3.3)$ & E148Q / M694V & $8(1.6)$ \\
P369S / - & $12(2.4)$ & E148Q / V726A & $7(1.4)$ \\
& & M680I / V726A & $6(1.2)$ \\
& & M694V / M680I & $4(0.8)$ \\
& & R761H / R761H & $4(0.8)$ \\
& & Others / Others & $4(0.8)$ \\
\end{tabular}

TABLE 5. Clinical features of homozygous or heterozygous specific mutations

\begin{tabular}{|c|c|c|c|c|}
\hline & \multicolumn{3}{|c|}{ Specific mutations } & \multirow{2}{*}{$\begin{array}{l}\text { Differences } \\
\text { (p) }\end{array}$} \\
\hline & $\begin{array}{c}\text { E148Q } \\
(n=228)\end{array}$ & $\begin{array}{l}M 694 V \\
(n=119)\end{array}$ & $\begin{array}{c}\text { Others } \\
(n=160)\end{array}$ & \\
\hline Age, years* & $8.9 \pm 3.7$ & $8.4 \pm 3.8$ & $8.5 \pm 3.7$ & a NS \\
\hline Age at onset, years* & $6.8 \pm 3.7$ & $4.9 \pm 3.0$ & $5.5 \pm 3.2$ & $\begin{array}{l}\text { a } 0.019, \mathrm{~b} 0.012, \\
{ }^{\mathrm{c}} 0.037, \mathrm{~d} \text { NS }\end{array}$ \\
\hline Age at diagnosis, years* & $8.9 \pm 3.6$ & $8.0 \pm 3.3$ & $7.9 \pm 3.1$ & ${ }^{\text {a }}$ NS \\
\hline Delay in diagnosis, years* & $2.1 \pm 2.4$ & $2.8 \pm 2.2$ & $2.4 \pm 2.3$ & ${ }^{a}$ NS \\
\hline Disease severity score* & $5.62 \pm 1.71$ & $6.84 \pm 2.10$ & $6.15 \pm 1.70$ & $\begin{array}{c}{ }^{\mathrm{a}} 0.004,{ }^{\mathrm{b}} 0.001, \\
{ }^{\mathrm{c}} \mathrm{NS},{ }^{\mathrm{d}} \mathrm{NS}\end{array}$ \\
\hline Fever (\%) & 90.9 & 93.0 & 99.0 & ${ }^{\mathrm{a}} \mathrm{NS}$ \\
\hline Abdominal pain (\%) & 97.5 & 98.8 & 98.2 & ${ }^{\mathrm{a}} \mathrm{NS}$ \\
\hline Arthralgia (\%) & 49.4 & 46.8 & 45.7 & ${ }^{\mathrm{a}} \mathrm{NS}$ \\
\hline Myalgia (\%) & 25.5 & 17.9 & 25.0 & ${ }^{a}$ NS \\
\hline Arthritis (\%) & 13.9 & 23.4 & 13.6 & ${ }^{\mathrm{a}} \mathrm{NS}$ \\
\hline Chest pain (\%) & 10.1 & 19.1 & 12.3 & ${ }^{a}$ NS \\
\hline Erysipelas-like erythema (\%) & 12.7 & 14.9 & 8.6 & ${ }^{\mathrm{a}} \mathrm{NS}$ \\
\hline \multicolumn{5}{|l|}{ Colchicine response } \\
\hline Complete response & $165(72.4)$ & $73(61.3)$ & $108(67.5)$ & \\
\hline Incomplete response & $59(25.9)$ & $37(31.1)$ & $45(28.1)$ & a NS \\
\hline No response & $4(1.7)$ & $9(7.6)$ & $7(4.4)$ & \\
\hline
\end{tabular}

* Mean \pm Standard deviation.

Differences: a. among three mutations groups, b. E148Q vs. M694V, c. E148Q vs. Others, d. M694V vs. Others.

NS: Not significant $(\mathrm{p}>0.05)$. 
and heterozygous $(5.9 \pm 1.9$ and $5.7 \pm 1.7$ points, respectively) $(p=0.007)$.

Patients were further divided into subgroups if they had one or two E148Q, M694V, or other mutated alleles. There were no significant differences in the clinical findings between all three subgroups ( $p>0.05$; Table 5$)$. Although, response to colchicine was found to be best in E148Q subgroup and the worst in M694V subgroup, the difference between subgroups did not reach to a statistically significant level $(p=0.062)$ (Table 5). However, significant differences were identified in mean age of symptoms onset and disease severity scores between the three subgroups $(p=0.008$ and $\mathrm{p}=0.001$, respectively).The M694V subgroup had the lowest age of disease onset and the highest disease severity scores, while the E148Q subgroup demonstrated an older mean age of disease onset with the lowest severity scores (Table 5).

\section{DISCUSSION}

Familial Mediterranean fever is a hereditary disease that is more prevalent in ethnics groups near the Mediterranean Sea. ${ }^{2}$ Particular mutations are more frequently observed in certain ethnic groups or geographic regions. For example, the M694V mutation has been more frequently identified in Turks and North African Jews, while the V726A mutation has been found to be more prevalent in Ashkenazi Jews, Armenians, and Arabs. ${ }^{1-2,5,7}$ Although, heterozygous patients tend to show relatively mild disease, heterozygous cannot be distinguished from homozygous FMF patients by disease severity. ${ }^{1}$
Significant interregional variations have been observed in studies investigating MEFV gene mutation frequencies throughout Turkey (Table 6). 3,5,9-16,20,21 The most common mutation characterized in Turks is the M694V mutation with the frequency of $30-50 \%$. The E148Q mutation occurs at a frequency ranging from $1.4-11.4 \%$ according to some previous studies. ${ }^{7}$ 15,16, 19-21 Although, there were disagreement regarding whether the E148Q mutation even exhibit disease, it has been reported as disease causing mutation in FMF. ${ }^{20}$ Two recent studies from eastern and southeast Turkey reported that the E148Q mutation occurs at frequencies of $34.1 \%$ and $30.7 \%$ in these populations, respectively, which is approximate to the frequency that we identified at $40.1 \% .^{3,13}$ A study from western Turkey, reported that the M694V mutation occurs most frequently at $48.6 \%$ and is followed by the E148Q, M680I and V726A mutations. ${ }^{9} \mathrm{~A}$ comprehensive study with adult and pediatric FMF patients from the entire country of Turkey also reported that the M694V mutation was the most frequent at $51.4 \%$, which was followed by M680I and V726A. ${ }^{5}$ Our study revealed a higher E148Q mutation frequency $(40.1 \%)$ than our previous study (30.7\%); however, the M694V frequency that we observed in present study is similar to our previous study $(25.9 \%$ versus $26.0 \%$, respectively). ${ }^{3}$ The differences between our first and second studies may be resulted from inclusion of more patients in the latter study.

Although some previous studies ${ }^{22,23}$ have proposed E148Q sequence variation as a polymorphism rather than a disease causing

TABLE 6. The most common MEFV mutations reported in different studies from the various regions of Turkey and some ethnic origins of other nationalities

\begin{tabular}{|c|c|c|c|c|c|c|c|c|}
\hline \multirow[t]{2}{*}{ Reference } & \multirow{2}{*}{$\begin{array}{c}\text { Geographic regions } \\
\text { of Turkey }\end{array}$} & \multirow{2}{*}{$\begin{array}{c}\text { Number of } \\
\text { patients }\end{array}$} & \multicolumn{6}{|c|}{ Mutations (\%) } \\
\hline & & & M694V & V726A & M680I & E148Q & R761H & P369S \\
\hline Tunca et al. ${ }^{5}$ & All over the Turkey & 1090 & 51.5 & 8.1 & 14.4 & NR & NR & NR \\
\hline Yalcinkaya et al. ${ }^{11}$ & Middle & 167 & 43.5 & 11.1 & 13.0 & NR & NR & NR \\
\hline Yesilada et al. ${ }^{12}$ & Middle & 420 & 28.2 & 5.3 & 15.7 & 34.1 & 5.2 & 6.5 \\
\hline Demirkaya et al. ${ }^{15}$ & Middle & 330 & 50.0 & 9.7 & 14.1 & 1.4 & 3.5 & NR \\
\hline Özdemir et al. ${ }^{13}$ & Middle- east & 3340 & 43.1 & 11.3 & 15.0 & 20.2 & 2.6 & 3.3 \\
\hline Yi it et al. ${ }^{14}$ & North & 625 & 33.9 & 4.9 & 15.8 & 3.5 & NR & NR \\
\hline Öztürk et al. ${ }^{20}$ & West & 452 & 51.9 & 10.8 & 10.4 & 15.5 & 1.0 & 5.5 \\
\hline Ece et al. ${ }^{3}$ & Southeast & 147 & 26.0 & 13.0 & 6.3 & 30.7 & 13.5 & 10.5 \\
\hline Present study & Southeast & 507 & 25.9 & 15.8 & 6.8 & 40.1 & 7.4 & 4.1 \\
\hline
\end{tabular}

NR: not reported. 
mutation, a more recent study ${ }^{24}$ have reported $85 \%$ of their patients who were homozygous for E148Q had typical FMF attacks. During attacks, acute phase reactants have been found raised and after attacks they returned to normal limits and these patients had a good response to colchicine treatment. In other two studies ${ }^{25,26} \mathrm{FMF}$ associated amyloidosis have been reported in patients who were heterozygous for E148Q and in apatient homozygous for both E148Q and V726A (E148Q V726A / E148Q V726A). Despite clinical heterogeneity, high frequency of symptoms in these previous studies are in accordance with our results.

Our results indicated that the M694V group demonstrates higher disease severity scores, while patients with the E148Q mutation have later disease onset with a milder disease course (Table 1). Previous studies also reported more severe disease phenotypes with the M694V mutation. ${ }^{3,20}$ In our study, patients with homozygous mutations showed greater disease severity and earlier disease onset when compared with heterozygous, which is in accordance with previous reports. ${ }^{1-3,5}$ Patients that are homozygous for the M694V mutation have a highly aggressive disease course and develop renal amyloidosis. ${ }^{1-3}$ The patients that we diagnosed with renal amyloidosis were either homozygous or compound heterozygous for the M694V mutation. In Turkey, FMF presents a heterogeneous genotype and disease course. Therefore, in our study, we found a low rate of homozygous mutations (approximately 14\%) and heterogeneous clinical findings despite high rate of parental consanguinity. In some previous studies from Turkey, in spite of high consanguineous marriages rates from all over the country, low homozygosity ratios (9-12\%) have been reported in FMF patients similar to our results. ${ }^{12,13}$ Other studies revealed that the E148Q mutation has been associated with milder FMF disease. ${ }^{3,20}$ Relatively mild FMF disease together with low disease severity scores were demonstrated in the majority of our patients, which may be due to the high frequency of E148Q mutations. ${ }^{1,3,20}$ No significant difference in diagnosis age among different genotype subgroups of our study, may be related to ineffective use of health services in some of our patients with low socio-economic status.

A previous study about symptoms associated with FMF attacks found that $80-94 \%$ of patients demonstrated fever, $55-91 \%$ had abdominal pain,
$21-84 \%$ complained of chest pain, and $27-70 \%$ had arthritis. ${ }^{2}$ In our study, we identified relatively lower rates of chest pain (13.8\%), and arthritis $(15.8 \%)$. Considering the high frequency of familial FMF history in our study, early diagnosis due to alertness of family might have led to easily diagnosing FMF without some variables such as chest pain and arthritis in these patients. It is known that, FMF may manifest only with fever in younger children and therefore diagnosis is most likely delayed in small children. ${ }^{27}$

In our study, regular and intermittent colchicine use was identified in $73.9 \%$ and $22.4 \%$ of patients, respectively. Complete response to treatment was observed in $68.3 \%$ of patients. It has been reported that response to colchicine may result in complete disappearance of attacks in $60-70 \%$ of patients, and $5 \%$ of patients are completely resistant to colchicine. ${ }^{28}$ It is possible that in our study intermittent colchicine use might be responsible for partial or no response to treatment.

Patient with AA-type amyloidosis and positive family history of FMF but without FMF clinical features are described as phenotype II. ${ }^{5}$ There were two patients with phenotype II in our study, both of them with homozygous mutations. This suggests that phenotype II is very rare in our FMF patients.

Highly consanguineous marriages are most common in the eastern and southeastern regions of Turkey. ${ }^{12,29}$ The mean rate of consanguineous marriages is $20.9 \%$ in overall Turkey, while this rate is $4.8 \%$ in western and $40.4 \%$ in eastern parts of the country. ${ }^{29}$ Therefore, our study sample also showed high parental consanguinity $(41.4 \%)$. An elevated degree of parental consanguinity may have contributed to the high rates of FMF family history, as autosomal recessive disorders tend to occur more frequently in consanguineous marriages.

Because Turkey historically located along immigration routes, it is nearly impossible to determine the exact ancestry of specific ethnic groups. Yet, inhabitants of southeastern Turkey are predominantly Kurdish and Arabic in origin. Differences in MEFV mutation gene pools between southeastern and other regions of Turkey may be due to southeastern Turkey's unique ethnic composition, and likely explains why there is a relatively high frequency of the E148Q mutation in this region. Our study and some previous reports suggest that E148Q mutation is related to $\mathrm{FMF},{ }^{19,30}$ since the frequency of E148Q 
is $6.5 \%$ in healthy Turkish population. ${ }^{30}$

In conclusion, the E148Q mutation is the most frequent $M E F V$ mutation in southeastern Turkey and is associated with milder disease phenotypes and later disease onset. This is in contrast to other regions of Turkey and the most areas of the world. This difference may be related to ethnic and geographic differences of this location.

\section{REFERENCES}

1. Marek-Yagel D, Berkun Y, Padeh S, Abu A, et al. Clinical disease among patients heterozygous for familial Mediterranean fever. Arthritis Rheum 2009;60(6):1862-6.

2. Ben-Chetrit E, Touitou I. Familial mediterranean Fever in the world. Arthritis Rheum 2009;61(10):1447-53.

3. Ece A, Çakmak E, Uluca Ü, Kelekçi S, et al. The MEFV mutations and their clinical correlations in children with familial Mediterranean fever in southeast Turkey. Rheumatol Int 2014;34(2):207-12.

4. Livneh A, Langevitz P, Zemer D, Zaks N, et al. Criteria for the diagnosis of familial Mediterranean fever. Arthritis Rheum 1997;40(10):1879-85.

5. Tunca M, Akar S, Onen F, Ozdogan H, et al. Familial Mediterranean fever (FMF) in Turkey: results of a nationwide multicenter study. Medicine (Baltimore) 2005;84(1):1-11.

6. Barron K, Athreya B, Kastner D. Periodic fever syndromes and other inherited autoinflammatory diseases. In: Cassidy JT, Petty RE, Laxer RM, Lindsley CB, eds. Textbook of Pediatric Rheumatology. $6^{\text {th }}$ ed. Philadelphia: Saunders Elsevier; 2011:642-60.

7. Ozen S, Demirkaya E, Amaryan G, Koné-Paut I, et al. Results from a multicentre international registry of familial Mediterranean fever: impact of environment on the expression of a monogenic disease in children. Ann Rheum Dis 2014;73(4):662-7.

8. Sarkisian T, Ajrapetyan H, Shahsuvaryan G. Molecular study of FMF patients in Armenia. Curr Drug Targets Inflamm Allergy 2005;4(1):113-6.

9. Coker I, Colak A, Yolcu I, Türkön H, H, et al. [MEFV gene mutation spectrum in familial Mediterranean fever (FMF): a single center study in the Aegean region of Turkey]. Z Rheumatol 2011;70(6):511-6. German.

10. Ureten K, GönülalanG,AkbalE, Güne F,etal.Demographic, clinical and mutational characteristics of Turkish familial Mediterranean fever patients: results of a single center in Central Anatolia. Rheumatol Int 2010;30(7):911-5.

11. Ertekin V, Selimo lu MA, Pirim I. Familial Mediterranean fever in a childhood population in eastern Turkey. Pediatr Int 2005;47(6):640-4.

12. Yalçinkaya F, Cakar N, Misirlio lu M, Tümer N, et al. Genotype-phenotype correlation in a large group of Turkish patients with familial mediterranean fever: evidence for mutation-independent amyloidosis. Rheumatology (Oxford) 2000;39(1):67-72.

13. Yesilada E, Taskapan H, Gulbay G. Prevalence of known mutations and a novel missense mutation (M694K) in the MEFV gene in a population from the Eastern Anatolia Region of Turkey. Gene 2012;511(2):371-4.
14. Ozdemir O, Sezgin I, Kurtulgan HK, Candan F, et al. Prevalence of known mutations in the MEFV gene in a population screening with high rate of carriers. Mol Biol Rep 2011;38(5):3195-200.

15. Yigit S, Bagci H, Ozkaya O, Ozdamar K, et al. MEFV mutations in patients with familial Mediterranean fever in the Black Sea region of Turkey: Samsun experience [corrected]. J Rheumatol 2008;35(1):106-13.

16. Demirkaya E, Tunca $Y$, Gok F, Ozen S, et al. A very frequent mutation and remarkable association of R761H with M694V mutations in Turkish familial Mediterranean fever patients. Clin Rheumatol 2008;27(6):729-32.

17. Moradian MM, Sarkisian T, Ajrapetyan H, Avanesian N. Genotype-phenotype studies in a large cohort of Armenian patients with familial Mediterranean fever suggest clinical disease with heterozygous MEFV mutations. J Hum Genet 2010;55(6):389-93.

18. Pras E, Livneh A, Balow JE Jr, Pras E, et al. Clinical differences between North African and Iraqi Jews with familial Mediterranean fever. Am J Med Genet 1998;75(2):216-9.

19. OzenS,Aktay N, Lainka E, Duzova A, et al. Disease severity in children and adolescents with familial Mediterranean fever: a comparative study to explore environmental effects on a monogenic disease. Ann Rheum Dis 2009;68(2):246-8.

20. Topaloglu R, Ozaltin F, Yilmaz E, Ozen S, et al. E148Q is a disease-causing MEFV mutation: a phenotypic evaluation in patients with familial Mediterranean fever. Ann Rheum Dis 2005;64(5):750-2.

21. Ozturk C,Halicioglu O, Coker I, Gulez N, et al. Association of clinical and genetical features in FMF with focus on MEFV strip assay sensitivity in 452 children from western Anatolia, Turkey. Clin Rheumatol 2012;31(3):493-501.

22. Ben-Chetrit E, Lerer I, Malamud E, Domingo C, et al. The E148Q mutation in the MEFV gene: is it a disease-causing mutation or a sequence variant? Hum Mutat 2000;15(4):3856.

23. Mimouni A, Magal N, Stoffman N, Shohat T, et al. Familial Mediterranean fever: effects of genotype and ethnicity on inflammatory attacks and amyloidosis. Pediatrics 2000;105(5):E70.

24. Topaloglu R, Ozaltin F, Yilmaz E, Ozen S, et al. E148Q is a disease-causing MEFV mutation: a phenotypic evaluation in patients with familial Mediterranean fever. Ann Rheum Dis 2005;64(5):750-2.

25. Akar N, Akar E, Yalçinkaya F. E148Q of the MEFV gene causes amyloidosis in familial Mediterranean fever patients. Pediatrics 2001;108(1):215.

26. Livneh A, Langevitz P, Shinar Y, Zaks N, et al. MEFV mutation analysis in patients suffering from amyloidosis of familial Mediterranean fever. Amyloid 1999;6(1):1-6.

27. Yalcinkaya F, Ozcakar ZB, Tanyildiz M, Elhan AH. Familial Mediterranean fever in small children in Turkey. Clin Exp Rheumatol 2011;29(4 Suppl 67):S87-90.

28. Ben-Chetrit E, Aamar S. About colchicine compliance, resistance and virulence. Clin Exp Rheumatol 2009;27(2 Suppl 53):S1-3.

29. Erdem $Y$, Tek en F. Genetic screening services provided in Turkey. J Genet Couns 2013;22(6):858-64.

30. Topaloglu R, Yildiz C, Taskiran E, Korkmaz E, et al. PW01004-The sequence analysis in E148Q homozygous patients. Pediatr Rheumatol Online J 2013;11(Suppl 1):A57. 